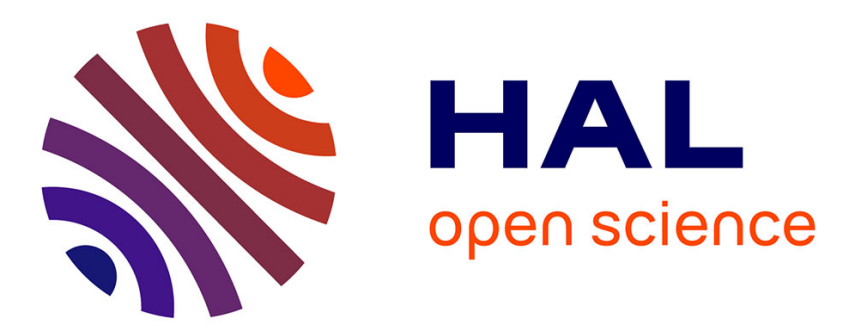

\title{
Étude d'une décharge à haute pression ionisée par un faisceau d'électrons à haute énergie \\ W.P. Allis
}

\section{To cite this version:}

W.P. Allis. Étude d'une décharge à haute pression ionisée par un faisceau d'électrons à haute énergie. Revue de Physique Appliquée, 1975, 10 (3), pp.97-103. 10.1051/rphysap:0197500100309700 . jpa00243902

\section{HAL Id: jpa-00243902 https://hal.science/jpa-00243902}

Submitted on 1 Jan 1975

HAL is a multi-disciplinary open access archive for the deposit and dissemination of scientific research documents, whether they are published or not. The documents may come from teaching and research institutions in France or abroad, or from public or private research centers.
L'archive ouverte pluridisciplinaire HAL, est destinée au dépôt et à la diffusion de documents scientifiques de niveau recherche, publiés ou non, émanant des établissements d'enseignement et de recherche français ou étrangers, des laboratoires publics ou privés. 


\title{
REVUE DE PHYSIQUE APPLIQUÉE
}

Supplément au "Journal de Physique *

\section{ÉTUDE D'UNE DÉCHARGE A HAUTE PRESSION IONISÉE PAR UN FAISCEAU D'ÉLECTRONS A HAUTE ÉNERGIE $\left({ }^{*}\right)$}

\author{
W. P. ALLIS
}

ONERA, 29, avenue de la Division-Leclerc, 92320 Châtillon, France

(Reçu le 14 octobre 1974, accepté le 3 février 1975)

\begin{abstract}
Résumé. - L'objectif ultime de cette étude est de connaître en détail la structure du plasma dans un laser à ionisation par faisceau d'électrons. Dans la première partie du travail présenté ici, nous considérons la partie ambipolaire $\left(n_{+}=n_{-}\right)$du plasma en tenant compte de la source uniforme $S$, de l'ionisation autonome $v_{\mathrm{i}} n$, de la recombinaison $\alpha n^{2}$, de l'attachement $\beta n$, de la diffusion $D_{\mathrm{a}} \Delta^{2} n$, et d'un courant appliqué $J$, tous les coefficients étant constants. Nous obtenons ainsi des formules fermées pour la densité électronique $n(x)$ et la caractéristique $V(J)$ de cette partie du plasma.

Dans les conditions usuelles pour les applications, le courant continu $J$ ne provient pas de la source $S$ mais de l'émission cathodique $\gamma J_{+}$. Nous développons dans la deuxième partie une théorie nouvelle de la chute cathodique qui ne repose pas sur le coefficient $\alpha_{i}$ de Townsend. Nous déduisons l'ionisation $i(V)$ du flux $\Phi(u, V)$ des électrons dans l'espace des énergies cinétique $u$ et potentielle $V(x)$. L'équation différentielle du premier ordre pour le flux $\Phi$ dépend des sections efficaces $Q(u)$ élastique, inélastiques, et d'ionisation, et comme celles-ci sont des données expérimentales, la résolution nécessite un ordinateur. Ceci est en préparation.
\end{abstract}

\begin{abstract}
The ultimate objective of this study is a detailed knowledge of the plasma structure in an electron beam ionization laser. In the first part we consider the ambipolar part $\left(n_{+}=n_{-}\right)$of the plasma, taking into account the uniform source $S$, the autonomous ionization $v_{1} n$, the recombination $\alpha n^{2}$, the attachment $\beta n$, the diffusion $D_{\mathrm{a}} \Delta^{2} n$, and an applied current $J$, all coefficients being constant. Thus, we obtain closed formulas for the electron density $n(x)$ and the characteristic $V(J)$ of this part of the plasma.

In the usual conditions for applications, the continuous current $J$ is not provided by the source $S$ but by the cathode emission $\gamma J_{+}$. In the second part we develop a new theory of the cathode drop which does not rest on the Townsend coefficient $\alpha_{i}$. We deduce the ionization $i(V)$ from the electron flux $\Phi(u, V)$ in the space of kinetic $u$ and potential $V(x)$ energies. The first order differential equation for the flux $\Phi$ depends on the elastic, unelastic and ionization cross sections $Q(u)$, and as these are experimental the resolution requires a computer. This is in preparation.
\end{abstract}

1. Introduction. - L'objectif du travail en cours, et dont nous présentons donc des résultats partiels, est de calculer en détail la distribution de la densité électronique $n_{-}$et du champ électrique $E$ dans une décharge à haute pression (pd $>100$ ) excitée par une source extérieure homogène $S$, telle que celle d'un laser $\mathrm{CO}_{2}$ à faisceau d'électrons [1]. Nous tenons compte des mobilités $\mu_{ \pm} E n_{ \pm}$, des diffusions $\nabla D_{ \pm} n_{ \pm}$, des flux $\Gamma_{ \pm}$, de l'ionisation autonome $n_{-} v_{\mathrm{i}}$, de l'attachement $\beta n_{-}$ (soit $v_{\mathbf{i}}$ négatif), et de la recombinaison $\alpha n_{+} n_{-}$.

(*) Etude sous contrat de la D.R.M.E.
Les équations correspondantes sont :

$$
\begin{array}{rlr}
\Gamma_{-}+\mu_{-} E n_{-} & =-\nabla D_{-} n_{-} & \\
\Gamma_{+}-\mu_{+} E n_{+} & = & -\nabla D_{+} n_{+} \\
\nabla . \Gamma_{ \pm}-v_{\mathrm{i}} n_{-} & =S^{\mathrm{AT}} & -\alpha n_{+} n_{-} \\
e n_{+} & =e n_{-}^{\mathrm{AT}} & +\varepsilon_{0} \nabla . E .
\end{array}
$$

Les termes de ces équations varient en ordre de grandeur dans diverses parties de la décharge [2] [3]. Il est 
donc utile pour bien comprendre la décharge, ainsi que pour effectuer les calculs, de la diviser en régions dites ambipolaire, transition, et chute cathodique, dans lesquelles les termes dominants sont différents. Les. termes écrits à gauche sont toujours retenus mais les termes à droite sont retenus seulement dans les régions indiquées au-dessus de chaque terme: C'est surtout dans l'éq. (3) que le changement est marqué, les termes de droite étant dominants dans la partie ambipolaire, ceux de gauche dans la partie cathodique, ainsi que dans l'éq. (4) dans laquelle on passe d'une région neutralisée à une région à charge positive. Nous présentons des résultats seulement théoriques pour les régions ambipolaire et cathodique et il n'y a pas encore de résultats numériques.

2. Région ambipolaire entre plans parallèles. 2.1. Solution ambipolaire. - Dans cette région, l'éq. (4) donne $n_{+}=n_{-}$. Posons $\Gamma_{+}=\Gamma_{-}$en remettant la prise en compte d'un courant continu $J$ à plus tard. Eliminons $E$ entre (1) et (2) et introduisons $\Gamma_{ \pm}$ dans (3). Nous obtenons ainsi l'équation pour la densité $n(x)$ :

$$
S+v_{\mathrm{i}} n=\alpha n^{2}-D_{\mathrm{a}} \nabla^{2} n
$$

En intégrant sur $\mathrm{d} n$ on obtient le gradient $g=\nabla n$ :

$$
\begin{aligned}
g^{2} & =\frac{2 \alpha}{3 D_{\mathrm{a}}}\left[n^{3}-n_{\mathrm{p}}^{3}-\frac{3}{2} \frac{v_{\mathrm{i}}}{\alpha}\left(n^{2}-n_{\mathrm{p}}^{2}\right)-3 \frac{S}{\alpha_{1}}\left(n-n_{\mathrm{p}}\right)\right] \\
& =\frac{2 \alpha}{3 D_{\mathrm{a}}}\left(n_{1}-n\right)\left(n_{\mathrm{p}}-n\right)\left(n-n_{2}\right)
\end{aligned}
$$

$g=0$ au maximum $n_{\mathrm{p}}$ de densité qui se trouve, pour $J=0$ à mi-chemin entre les plans; mais il y a deux autres solutions $n_{1}>n_{\mathrm{p}}$ et $n_{2}<0$ pour lesquelles $g=0$.

Introduisons les symboles

$$
\begin{aligned}
n_{\mathrm{p}}^{\prime} & =n_{\mathrm{p}}-v_{\mathrm{i}} / 2 \alpha \\
\delta & =\frac{S+v_{\mathrm{i}} n_{\mathrm{p}}-\alpha n_{\mathrm{p}}^{2}}{\alpha}>0 ;
\end{aligned}
$$

$\delta$ mesure les pertes par diffusion car il mesure le manque d'équilibre au centre entre l'ionisation et la recombinaison. Nous nous intéressons aux $\delta$ petits. Le discriminant de l'équation quadratique pour $n_{1}, n_{2}$ est alors

$$
\begin{aligned}
\sigma^{2} & =\left(\frac{3 v_{\mathrm{i}}}{2 \alpha}\right)^{2}+3 \frac{4 S+v_{\mathrm{i}} n_{\mathrm{p}}-\alpha n_{\mathrm{p}}^{2}}{\alpha} \\
& =9 n_{\mathrm{p}}^{\prime 2}+12 \delta
\end{aligned}
$$

et

$$
2 n_{1,2}=-n_{\mathrm{p}}^{\prime}+\frac{v_{\mathrm{i}}}{\alpha} \pm \sigma
$$

On intègre alors l'éq. (6) pour obtenir

$$
\begin{aligned}
\xi & =\left(\frac{2 \alpha \sigma}{3 D_{\mathrm{a}}}\right)^{1 / 2} x \\
= & \sigma^{1 / 2} \int_{n}^{n_{0}} \frac{\mathrm{d} n}{\left[\left(n_{1}-n\right)\left(n_{\mathrm{p}}-n\right)\left(n-n_{2}\right)\right]^{1 / 2}} \\
& \frac{n_{1}-n_{\mathrm{p}}}{n_{1}-n}=d n^{2}(\xi / 2) ;
\end{aligned}
$$

$d n(\xi / 2)$ est une des fonctions elliptiques de Jacobi de module

$$
m=\frac{n_{\mathrm{p}}-n_{2}}{n_{1}-n_{2}}=\frac{\sigma+3 n_{\mathrm{p}}^{\prime}}{2 \sigma}
$$

et de module complémentaire

$$
m_{1}=\frac{n_{1}-n_{\mathrm{p}}}{n_{1}-n_{2}}=\frac{\sigma-3 n_{\mathrm{p}}^{\prime}}{2 \sigma} .
$$

Ici, comme dans (10), il faut prendre $\sigma>0$.

Nous pouvons maintenant transformer la fonction $d n^{2}(\xi / 2)$ pour obtenir

$$
n_{\mathrm{p}}-n=\frac{3 \delta}{\sigma} \frac{1-c n(\xi)}{d n(\xi)+m c n(\xi)+m_{1}}
$$

et

$$
\frac{\mathrm{d} n}{\mathrm{~d} \xi}=-\frac{3 \delta}{\sigma} \frac{\operatorname{sn}(\xi)}{\operatorname{dn}(\xi)+m c n(\xi)+m_{1}}
$$

La densité centrale $n_{\mathrm{p}}$ est déterminée par les conditions aux bords

$$
\begin{aligned}
x & = \pm L / 2 \\
\xi_{0}^{2} & =\alpha \sigma L^{2} / 6 D_{\mathrm{a}} \\
n_{\mathrm{p}} & =\frac{3 \delta}{\sigma} \frac{1-\operatorname{cn}\left(\xi_{0}\right)}{\operatorname{dn}\left(\xi_{0}\right)+\operatorname{mcn}\left(\xi_{0}\right)+m_{1}} .
\end{aligned}
$$

Cette équation est d'ailleurs implicite car $\sigma$ et $\delta$ dépendent de $n_{\mathrm{p}}$. Il est donc utile de chercher les expressions limites plus simples des fonctions elliptiques.

Notons d'abord une situation particulière.

Pour

et

$$
\begin{aligned}
& v_{\mathrm{i}}=2 \alpha n_{\mathrm{p}}, \\
& n_{\mathrm{p}}=0, \quad 2 n_{\mathrm{p}}=n_{1}+n_{2} \\
& m=m_{1}=\frac{1}{2} .
\end{aligned}
$$

Cette condition n'apporte pas de simplification, mais marque la transition entre les limites hyperbolique et trigonométrique que nous allons étudier.

2.2 Limite PARAboliQue. - Considérons d'abord le cas où tous les $\xi$ sont petits :

$$
\xi_{0}^{2}=\frac{\alpha \sigma L^{2}}{6 D_{\mathrm{a}}} \rightarrow 0,
$$

ce qui correspond à la prédominance de la diffusion. Les fonctions elliptiques peuvent alors être remplacées 
par des séries en puissances de $\xi^{2}$ et il vient en première approximation

$$
\begin{aligned}
& n_{\mathrm{p}}=\frac{\alpha \delta}{8 D_{\mathrm{a}}} L^{2}=\frac{S+v_{\mathrm{i}} n_{\mathrm{p}}-\alpha n_{\mathrm{p}}^{2}}{8 D_{\mathrm{a}}} L^{2} \\
& n=n_{\mathrm{p}}\left(1-4 x^{2} / L^{2}\right) .
\end{aligned}
$$

C'est une distribution parabolique.

2.3 LIMITE HYPERBOLIQUE. - La limite inverse est celle où la diffusion est petite avec équilibre local : $\delta \rightarrow 0$ d'où $n_{\mathrm{p}}^{\prime}>0$. On trouve alors

$$
\begin{aligned}
\sigma & =3 n_{\mathrm{p}}^{\prime}+2 \delta / n_{\mathrm{p}}^{\prime} \\
n_{1} & =n_{\mathrm{p}}+\delta / n_{\mathrm{p}}^{\prime} \\
m_{1} & =2 \delta / 3 n_{\mathrm{p}}^{\prime 2} \rightarrow 0 .
\end{aligned}
$$

Les racines $n_{1}$ et $n_{\mathrm{p}}$ se confondent, et l'intégrale (10) conduit à des fonctions hyperboliques.

$$
\begin{aligned}
& \operatorname{cn}(\xi) \rightarrow \operatorname{dn}(\xi) \rightarrow \operatorname{sech}(\xi) \\
& \operatorname{sn}(\xi) \rightarrow \tanh (\xi) .
\end{aligned}
$$

Il vient

$$
\begin{aligned}
n_{\mathrm{p}}-n & =\frac{\delta}{2 n_{\mathrm{p}}^{\prime}}(\cosh \xi-1) \\
\cosh \xi_{0} & =\frac{S+\alpha n_{\mathrm{p}}^{2}}{S+v_{\mathrm{i}} n_{\mathrm{p}}-\alpha n_{\mathrm{p}}^{2}} \rightarrow \infty .
\end{aligned}
$$

Ces formules montrent bien l'aplatissement du centre quand $\delta / n_{\mathrm{p}}^{\prime} \rightarrow 0$ et le raidissement des bords quand $\xi_{0} / L \rightarrow \infty$.

2.4 Limite TRIGONOMÉTRIQUE. - La limite opposée à la précédente est celle où $\alpha \rightarrow 0$, donc $n_{\mathrm{p}}^{\prime}<0$. Il vient alors

$$
\begin{aligned}
& \sigma=\frac{3 v_{\mathrm{i}}}{2 \alpha}+\frac{4 S}{v_{\mathrm{i}}}+n_{\mathrm{p}} \rightarrow \infty \\
& n_{1}=\frac{3 v_{\mathrm{i}}}{2 \alpha}+\frac{2 S}{v_{\mathrm{i}}} \rightarrow \infty \\
& m=2 \frac{S+v_{\mathrm{i}} n_{\mathrm{p}}}{v_{\mathrm{i}} \sigma} \rightarrow 0
\end{aligned}
$$

et les fonctions elliptiques deviennent donc trigonométriques.

$$
\begin{gathered}
\operatorname{sn}(\xi) \rightarrow \sin (\xi), \quad c n(\xi) \rightarrow \cos (\xi), \\
d n(\xi) \rightarrow 1 .
\end{gathered}
$$

On trouve alors

$$
\begin{aligned}
\cos \xi_{0} & =\frac{S}{S+v_{\mathbf{i}} n_{\mathrm{p}}} . \\
n & =\frac{S}{v_{\mathbf{i}}}\left(\frac{\cos \xi}{\cos \xi_{0}}-1\right) .
\end{aligned}
$$

On remarque qu'en général $\xi_{0}<\pi / 2$ mais que si

$$
\begin{aligned}
& S \ll v_{i} n_{\mathrm{p}}, \\
& \xi_{0} \rightarrow \pi / 2,
\end{aligned}
$$

et on retrouve la forme normale d'une décharge autonome. Par contre, si

$$
\begin{aligned}
v_{\mathrm{i}} n_{\mathrm{p}} & \ll S, \\
\xi_{0} & \rightarrow 0
\end{aligned}
$$

et on retombe sur un cas particulier de la limite parabolique.

2.5 Champ ambipolaire. - Ayant calculé la densité du plasma $n(x)$, on connaît le flux ambipolaire

$$
\Gamma_{\mathrm{a}}=-\nabla D_{\mathrm{a}} n,
$$

le champ ambipolaire

$$
E_{\mathrm{a}}=\frac{D_{+}-D_{-}}{\mu_{+}+\mu_{-}} \frac{\nabla n}{n}
$$

et le potentiel $V$ par rapport au centre, est donné par

où

$$
n=n_{\mathrm{p}} \exp \left(V / T_{\mathrm{a}}\right)
$$

$$
T_{\mathrm{a}}=\frac{D_{-}-D_{+}}{\mu_{-}+\mu_{+}}<T_{\mathrm{e}} .
$$

Le potentiel $V$ est négatif et les électrons ont une distribution de Boltzmann dans ce potentiel quoique à une température $T_{\mathrm{a}}$ légèrement inférieure à $T_{\mathrm{e}}$.

Comme $V=-\infty$ pour $n=0$, il est évident que la distribution ambipolaire n'est pas valable jusqu'à ce point. Il faut la couper à un certain potentiel $V_{0}$ correspondant à une certaine densité $n_{0}$.

Cette densité $n_{0}$ est déterminée par la condition que le flux d'une distribution en vitesse $\mathbf{v}$ initialement isotrope mais qui est coupée par une surface complètement absorbante est donnée par $n \bar{v} / 4$. Donc

et

$$
\begin{gathered}
n_{0}=\left(2 \pi m / e T_{\mathrm{e}}\right)^{1 / 2} \Gamma_{-} \\
V_{0}=T_{\mathrm{a}} \log \left(\frac{\Gamma_{-}}{n_{\mathrm{p}}} \sqrt{\frac{2 \pi m}{e T_{\mathrm{e}}}}\right) .
\end{gathered}
$$

2.6 SUPERPoSITION D'UN COURANT CONTINU. - $\mathrm{Si}$ on fait passer un courant $J$ homogène, les flux seront changés :

$$
\begin{aligned}
& \Gamma_{+}=\Gamma_{\mathrm{a}} \pm \frac{\mu_{+}}{\mu_{-}+\mu_{+}} \frac{J}{e}, \\
& \Gamma_{-}=\Gamma_{\mathrm{a}} \mp \frac{\mu_{-}}{\mu_{-}+\mu_{+}} \frac{J}{e},
\end{aligned}
$$

le signe supérieur s'appliquant du côté cathode et les flux étant positifs vers les surfaces. Comme la divergence de $J$ est nulle, l'équation fondamentale (5) n'est pas changée. Tout ce qui a été calculé en volume n'est 
donc pas changé. On ajoute simplement les flux additionnels aux flux ambipolaires et le champ ohmique,

$$
E_{J}=J /\left(\mu_{-}+\mu_{+}\right) n e,
$$

au champ ambipolaire (28).

Les conditions aux limites sont pourtant changées. Le flux électronique vers la cathode étant diminué, le potentiel de la cathode $V_{\mathrm{c}}<V_{0}$ descend et celui de l'anode monte, $V_{\mathrm{a}}>V_{0}$. Finalement, le courant appliqué domine à l'anode et on a :

$$
V_{\mathrm{a}}=T_{\mathrm{a}} \log \left(\frac{J}{e n_{\mathrm{p}}} \sqrt{\frac{m}{2 \pi e T_{\mathrm{e}}}}\right),
$$

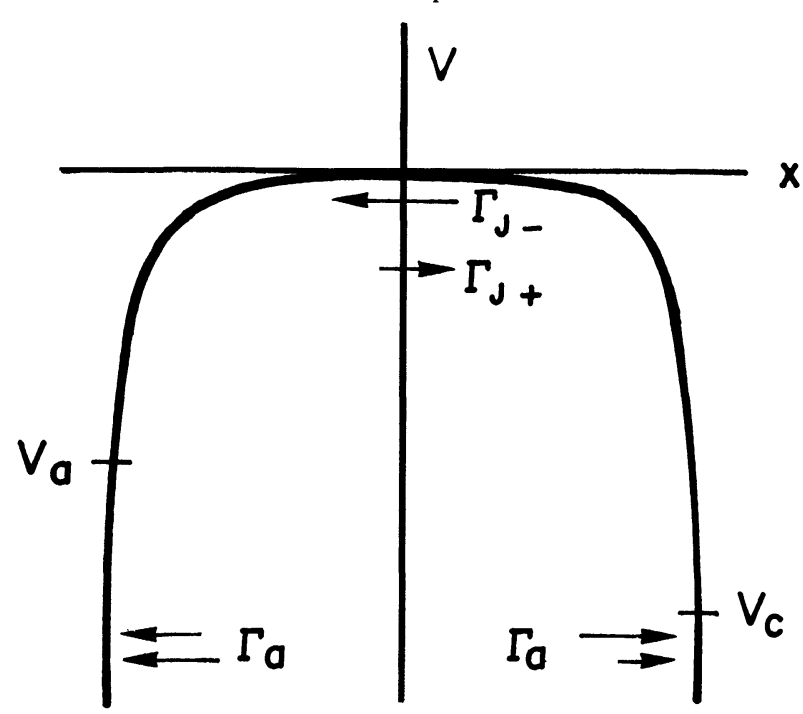

Fig. 1. - Distribution de potentiel et direction des flux sans émission secondaire à la cathode.

auquel on doit ajouter le potentiel ohmique.

Le potentiel ohmique entre le centre et l'anode se calcule au moyen de la transformation suivante :

$$
\begin{aligned}
V_{J}= & \int \frac{J / e}{\mu_{+}+\mu_{-}} \frac{\mathrm{d} x}{n}=\frac{J / e}{\mu_{+}+\mu_{-}} \int_{n_{\mathrm{a}}}^{n_{\mathrm{p}}} \frac{\mathrm{d} n}{n g} \\
= & \frac{J / e}{\mu_{+}+\mu_{-}}\left(\frac{3 D_{\mathrm{a}}}{2 \alpha}\right)^{1 / 2} \times \\
& \quad \times \int_{n_{\mathrm{a}}}^{n_{\mathrm{p}}} \frac{\mathrm{d} n}{n\left[\left(n_{1}-n\right)\left(n_{\mathrm{p}}-n\right)\left(n-n_{2}\right)\right]^{1 / 2}} .
\end{aligned}
$$

En remarquant que quand $\delta \rightarrow 0, n_{1} \rightarrow n_{\mathrm{p}}+\delta / n_{\mathrm{p}}^{\prime}$, on a

$$
\left(n_{1}-n\right)\left(n_{\mathrm{p}}-n\right)=\left(n_{\mathrm{p}}-n+\delta / 2 n_{\mathrm{p}}^{\prime}\right)^{2}-\left(\delta / 2 n_{\mathrm{p}}^{\prime}\right)^{2}
$$

et le dernier terme peut être négligé. L'intégrale s'effectue alors facilement et on trouve :

$$
\begin{aligned}
V_{J}=\frac{J / e}{\mu_{+}}+\mu_{-} & \frac{1}{n_{\mathrm{p}}}\left(\frac{3 D_{\mathrm{a}}}{2 \alpha n_{\mathrm{p}}^{\prime}}\right)^{1 / 2} \times \\
& \times\left[\frac{1}{\sqrt{2}} \log \frac{\left[\left(2 n_{\mathrm{p}}^{\prime}+n_{\mathrm{a}}\right)^{1 / 2}+\left(2 n_{\mathrm{p}}^{\prime}\right)^{1 / 2}\right]^{2}}{(\sqrt{3}+\sqrt{2})^{2} n_{\mathrm{a}}}\right. \\
& +\frac{1}{\sqrt{3}} \log \frac{6 n_{\mathrm{p}}^{\prime}}{\left[\left(2 n_{\mathrm{p}}^{\prime}+n_{\mathrm{a}}\right)^{1 / 2}+\left(3 n_{\mathrm{p}}^{\prime}\right)^{1 / 2}\right]^{2}} \\
& \left.+\frac{1}{\sqrt{3}} \log 4 n_{\mathrm{p}}^{\prime} \frac{n_{\mathrm{p}}-n_{\mathrm{a}}}{\delta}\right] .
\end{aligned}
$$

Les deux premiers termes dépendent de $n_{\mathrm{p}}^{\prime} / n_{\mathrm{a}}$ et non de la longueur du plasma. Ils représentent la résistance du plasma peu dense près de l'anode.

Dans le dernier terme, on $a$, d'après (22),

$$
4 n_{\mathrm{p}}^{\prime}\left(n_{\mathrm{p}}-n_{\mathrm{a}}\right) / \delta=2\left(\cosh \xi_{\mathrm{a}}-1\right) \rightarrow \exp \left(\xi_{\mathrm{a}}\right) .
$$

En prenant l'exponentielle, le dernier terme $V_{J 3}$ devient

$$
V_{J 3}=\frac{J / e}{\mu_{+}+\mu_{-}} \frac{L}{2 n_{\mathrm{p}}}
$$

qui correspond exactement à la résistance d'un plasma $n_{\mathrm{p}}$ plat. Si l'on retient le $\cosh \xi_{\mathrm{a}}$ on obtient l'effet de la courbure du plasma, mais les gaines sont toujours données par $V_{J 1}$ et $V_{J 2}$.

Les choses ne sont pas si simples à la cathode car le flux $\Gamma_{-}$tend vers zéro et le potentiel $V_{\mathrm{c}}$ vers $-\infty$. Lorsque $V_{\mathrm{c}}$ dépasse quelques centaines de volts les ions produisent une émission secondaire $\Gamma_{-}=-\gamma \Gamma_{+}$, ce qui permet au flux électronique de changer de signe sans que $V_{c}$ passe par l'infini. Mais ce processus est instable. Il y a un changement brusque entre la chute cathodique uniforme à haute tension et la chute dite normale, à plus basse tension mais plus grande densité de courant et qui ne recouvre donc qu'une partie de la surface cathodique.

Il y a une instabilité semblable à l'anode, mais à un courant $\mu_{-} / \mu_{+}$fois plus grand, quand le courant ionique tend vers zéro. Il se forme des taches anodiques où les ions supplémentaires sont produits dans le gaz.

3. La chute cathodique $[4,5]$. -3.1 LES COEFFCIENTS D'IONISATION. - On définit trois coefficients d'ionisation par les relations [2]

$$
\mathrm{d} n / n=v_{\mathrm{i}} \mathrm{d} t=\alpha_{\mathrm{i}} \mathrm{d} x=\eta_{\mathrm{i}} \mathrm{d} V ;
$$

l'ionisation par volt de parcours est donc

$$
\eta_{\mathrm{i}}=\alpha_{\mathrm{i}} / E=v_{\mathrm{i}} / E v_{\mathrm{d}}=v_{\mathrm{i}} / \mu_{-} E^{2} .
$$

Ces définitions s'appliquent généralement à l'ensemble des électrons dans un plasma homogène et on montre que ces coefficients sont proportionnels à $p$ et sont fonctions de $E / p$. Ces fonctions ont été appliquées directement à la chute cathodique [3], faute de mieux, car on ne connaissait ni la fonction $f(u)$ de distribution en énergie cinétique $u$ des électrons, ni un nombre suffisant des sections efficaces $Q_{\mathrm{i}}(u), Q_{k}(u), Q_{\mathrm{e}}(u)$ d'ionisation, d'excitation du niveau $k$, et élastique de transfert de quantité de mouvement. Nous nous proposons de calculer la distribution $f(u, x)$ sous forme de flux $\Phi(u, V)$ d'électrons dans l'espace des énergies $(u, V)$ ou $(u, \mathrm{~W})$ [6], où $W$ représente l'énergie totale d'un électron :

$$
W=u+V .
$$

Le signe de $V$ tient compte de la charge négative de l'électron. 
Pour une distribution monoénergétique,

$$
\begin{aligned}
& \mu_{-}=\frac{e}{m v Q_{\mathrm{e}} N}, \\
& \eta_{\mathrm{i}}=\frac{2 u Q_{\mathrm{e}}(u) Q_{\mathrm{i}}(u)}{(E / N)^{2}}=\frac{H_{\mathrm{i}}(u)}{\bar{V}_{k}(E / p)^{2}}, \\
& \eta_{x}=\frac{2 u Q_{\mathrm{e}} \sum Q_{k}(u)}{(E / N)^{2}}=\frac{H(u)}{\bar{V}_{k}(u)(E / p)^{2}} ;
\end{aligned}
$$

$\eta_{x}$ représente toutes les collisions inélastiques, y compris les ionisations, par volt de parcours et $\bar{V}_{k}(u) \eta_{x}$ représente l'énergie moyenne perdue par volt de parcours, y compris par collisions élastiques si celles-ci ne sont pas négligeables.

Nous supposons que $(E / p)^{2}$, que nous écrirons $E_{p}^{2}$, est connu en fonction de $V$. Nous savons d'avance que cette relation est approximativement linéaire, car l'expérience montre que $E_{p}$ est linéaire en $p x$ [2], mais on finit par calculer $E_{p}^{2}(V)$, ce qui permet de corriger l'hypothèse initiale.

3.2 L'ÉQUATION DU FLUX. - Considérons le flux $\Phi$ dans l'espace $(W, V)$. En absence de collisions les électrons se déplaceraient sur une ligne $W=$ Cte vers les $V$ décroissants.

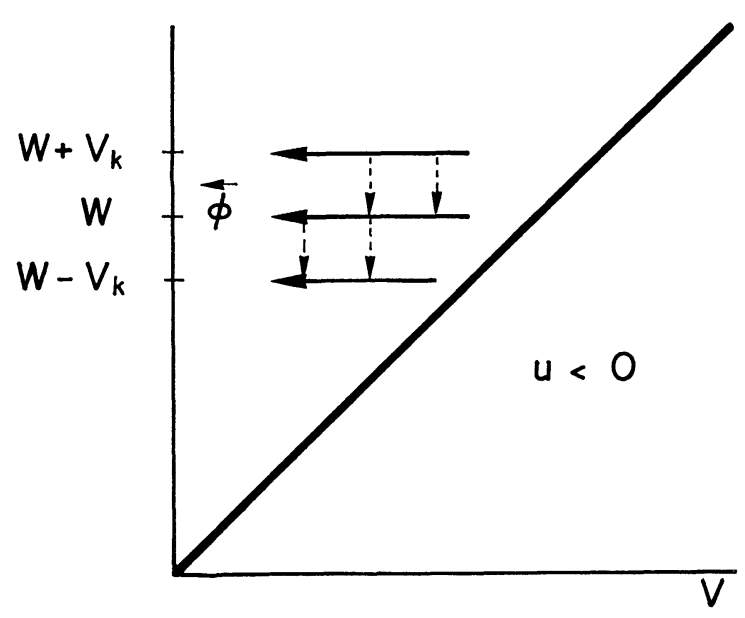

Fig. 2. - Direction du flux $\Phi$ dans l'espace des énergies $W, V$.

En présence de collisions élastiques, ils se déplacent aussi sur $W=\mathrm{Cte}$, mais avec un mouvement de va-et-vient. Le flux $\Phi$ est quand même constant et vers les $V$ décroissants. A cause des collisions inélastiques le flux $\Phi(W)$ reçoit en fait des contributions $\eta_{x}\left(W+\overline{V_{k}}\right) \Phi\left(W+\bar{V}_{k}\right)$ des lignes de flux supérieures à $W$ et perd $\eta_{x}(W) \Phi(W)$ vers les lignes de flux inférieures. La somme sur $k$ que nous avons écrite dans (6) devrait porter sur $\Phi\left(W+V_{k}\right)$ mais nous avons pris une moyenne $\bar{V}_{k}$.

$$
\begin{aligned}
-\frac{\partial \Phi}{\partial V} & =\eta_{x}\left(W+\bar{V}_{k}\right) \Phi\left(W+\bar{V}_{k}\right)-\eta_{x}(W) \Phi(W) \\
& =\bar{V}_{k} \frac{\partial \eta_{x} \Phi}{\partial W} .
\end{aligned}
$$

La chute cathodique étant d'environ $300 \mathrm{~V}$ et les potentiels d'excitation moins de $16 \mathrm{~V}$, nous pouvons remplacer la différence par la dérivée pourvu que l'énergie $u$ ne soit pas trop voisine d'un potentiel d'excitation $V_{k}$. Si on définit le vecteur $\Phi$ par

$$
\begin{aligned}
& \Phi_{V}=-\Phi \\
& \Phi_{W}=-\bar{V}_{k} \eta_{k} \Phi
\end{aligned}
$$

on a l'équation de conservation

$$
\nabla . \Phi=0
$$

Il est pourtant plus utile, bien que moins élégant du point de vue de l'écriture, de conserver le scalaire $\Phi$, mais en remplaçant les variables $(\mathrm{W}, V)$ par $(u, V)$. L'éq. (7) s'écrit alors

$$
E_{p}^{2} \frac{\partial \Phi}{\partial V}+\left(H-E_{p}^{2}\right) \frac{\partial \Phi}{\partial u}+\Phi \frac{\mathrm{d} H}{\mathrm{~d} u}=0
$$

3.3 LES CARACTÉRISTIQUES. - Les équations des caractéristiques de (10) sont

$$
\frac{\mathrm{d} V}{E_{p}^{2}}=\frac{\mathrm{d} u}{H-E_{p}^{2}}=\frac{-\mathrm{d} \Phi}{\Phi} \frac{\mathrm{d} u}{\mathrm{~d} H} ;
$$

ce sont les lignes de flux du vecteur (8).

Ces équations sont difficiles à intégrer à cause du terme $H(u)-E_{p}^{2}(V)$, mais on peut considérer les limites. Près de la cathode $E_{p}^{2}$ est grand et pour les basses énergies cinétiques $H(u)$ est petit.

a) Pour $E_{p}^{2} \gg H$,

$$
\begin{aligned}
& \mathrm{d} V+\mathrm{d} u=\mathrm{d} W=0 \\
& \Phi=\Phi(W) .
\end{aligned}
$$

Les pertes sont négligeables. Les lignes de flux sont à énergie totale $W$ constante et $\Phi$ est une fonction arbitraire de $W$. L'énergie cinétique des électrons augmente.

b) Pour

$$
H(u)=E_{\mathrm{p}}^{2}(V), \quad \mathrm{d} u=0 .
$$

L'énergie des électrons est maximale sur la courbe $H(u)=E_{p}^{2}(V)$. Près de la lueur négative, le champ $E_{p}^{2}$ est petit. Alors

c) Pour $E_{p}^{2} \ll H$, on définit les fonctions

$$
\begin{aligned}
& K(u)=\int_{u}^{V_{\mathrm{c}}} \frac{\mathrm{d} u}{H(u)} \\
& \Lambda(V)=\int_{V}^{V_{\mathrm{c}}} \frac{\mathrm{d} V}{E_{p}^{2}(V)} .
\end{aligned}
$$


Les lignes de flux sont alors données par

et

$$
K(u)=\Lambda(V)+\text { const }
$$

$$
H(u) \Phi=\psi(K-\Lambda),
$$

où $\psi$ est une fonction arbitraire de $K(u)-\Lambda(V)$.

C'est seulement dans le cas particulier où

$$
H(u)=a u
$$

que nous avons trouvé l'équation générale pour les lignes de flux

$$
u=\mathrm{e}^{-a \Lambda} \int_{V}^{\mathrm{c}} \mathrm{e}^{a \Lambda} \mathrm{d} V
$$

Les lignes de flux calculées par cette équation avec $E_{p}^{2}=2 a V$ sont tracées sur la figure suivante

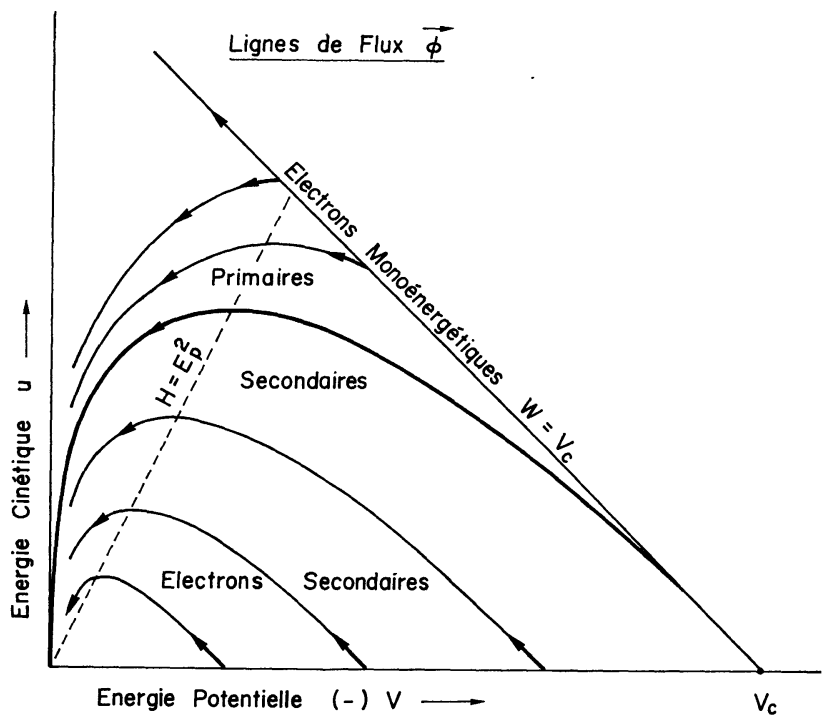

FIG. 3. - Lignes de flux $\Phi$ dans l'espace des énergies $u, V$.

3.4 LES ÉLECTRONS PRIMAIRES. - Soit $J_{00}$ l'émission cathodique et $J_{0}$ le courant d'électrons n'ayant pas eu de collision inélastique, et donc monoénergétique : $W=V_{\mathrm{c}}$. Ce courant est donné $[7,8]$ par

$$
\begin{gathered}
J_{0}=J_{00} \exp [-\xi(V)] \quad \xi(V)=\int_{V}^{V_{c}} \eta_{x} \mathrm{~d} V \\
u=V_{\mathrm{c}}-V .
\end{gathered}
$$

Les électrons qui ont subi une collision inélastique entrent dans le flux primaire $\Phi_{1}$, avec

$$
\left.\begin{array}{l}
\Phi_{1 V}=-J_{0} / \bar{V}_{k} \\
\Phi_{1 W}=-\eta_{x} J_{0}
\end{array}\right\} \quad \text { sur } \quad u=V_{\mathrm{c}}-V
$$

C'est la condition nécessaire pour définir la solution de (9) et (10) et le flux primaire correspondant remplit sur le diagramme $u-V$ l'espace entre la droite $u=V_{\mathrm{c}}-V$ et la caractéristique critique $u=u_{\mathrm{c}}(V)$ qui passe par $u=0, V=V_{\mathrm{c}}$.

Les électrons primaires produisent une ionisation

$$
i_{1}(V)=J_{0} \eta_{\mathrm{i}}+\int_{u_{\mathrm{c}}}^{V_{\mathrm{c}}-V} \eta_{\mathrm{i}} \Phi_{1} \mathrm{~d} u .
$$

3. 5 LES ÉLECTRONS SECONDAIRES, etc... - Nous supposons que les électrons secondaires sont libérés sans énergie cinétique, donc sur la ligne $u=0$. En calculant $\bar{V}_{k}$ nous supposons que l'électron primaire perd exactement $V_{\mathrm{i}}$, de sorte que l'énergie totale est correctement calculée. Il en résulte une source

$$
\Phi_{2 V}=i_{1}(V) \text { sur } u=0 \text { au point } V
$$

car $\Phi_{W}=0$ sur $u=0$. La solution de l'éq. (10) définie par cette source occupe la partie du plan $u-V$ en dessous de la caractéristique critique. Elle produit une ionisation

$$
i_{2}(V)=\int_{0}^{u_{\mathrm{c}}} \eta_{\mathrm{i}} \Phi_{2} \mathrm{~d} u
$$

qui est la source de la troisième génération

$$
\Phi_{3 V}=i_{2}(V) \text { sur } u=0
$$

et ainsi de suite.

La série des $i_{n}(V)$ converge parce que chaque flux $\Phi_{n}$ est décalé de $-V_{\mathrm{i}}$ vers $V=0$ et les énergies moyennes diminuent. Elle se termine à $n_{\mathrm{c}}=V_{\mathrm{c}} / V_{\mathrm{i}}$. On peut alors calculer la densité d'ionisation totale

$$
i(V)=\sum_{1}^{n_{\mathrm{c}}} i_{n}(V)
$$

et le courant électronique total

$$
\begin{aligned}
& J_{-}(V)=J_{00}+J_{2}(V), \\
& J_{2}(V)=\int_{V}^{V_{c}} i(V) \mathrm{d} V .
\end{aligned}
$$

L'ionisation par volt de parcours est donnée par

$$
\eta_{\text {eff }}=i(V) / J_{-}(V)
$$

et pour un champ uniforme, ce coefficient ne peut être supérieur à $1 / V_{\mathrm{i}}$. En fait, il a un maximum d'environ $1 / 3 V_{\mathrm{i}}$ à $E_{p} \approx 300$ sauf pour les mélanges Penning où il atteint facilement $2 / 3 V_{\mathrm{i}}$. Nous pensons que dans nos calculs $\eta_{\text {eff }}(V)$ sera presque nul près de la cathode et bien supérieur à $1 / V_{\mathbf{i}}$ vers la fin de la chute cathodique.

Appelons $V_{1}$ le potentiel du point de séparation du courant ionique, c'est-à-dire le point tel que le courant ionique arrivant à la cathode soit égal à l'ionisation totale entre $V_{\mathrm{c}}$ et $V_{1}$. Ce n'est pas nécessairement le point où $E_{p}^{2}=0$ et s'il y a de l'ionisation plus loin de la cathode, ces ions disparaissent par recombinaison ou par diffusion latérale. Ce point est à l'intérieur de la lueur négative. On a alors pour l'émission secondaire à la cathode

$$
J_{00}=\gamma J_{+}\left(V_{\mathrm{c}}\right)=\gamma J_{2}\left(V_{1}\right) .
$$

$J_{2}$ étant proportionnel à $J_{00}$, c'est une relation entre $\left(V_{c}-V_{1}\right)$ et $\gamma$ qui varient dans le même sens. 
Le courant ionique entre $V_{1}$ et $V_{\mathrm{c}}$ est donné par

$$
J_{+}(V)=J_{2}\left(V_{1}\right)-J_{2}(V)
$$

et l'équation de Poisson s'écrit

$$
\frac{J_{+}}{p^{2} v_{d+}}-\frac{J_{-}}{p^{2} v_{d-}}=\frac{1}{2} \varepsilon_{0} \frac{\mathrm{d} E_{p}^{2}}{\mathrm{~d} V} .
$$

Dans la chute cathodique même, la charge d'espace des électrons est négligeable et l'équation se simplifie en :

$$
J_{+}(V)=\frac{\varepsilon_{0}}{2} p^{2} v_{d+} \frac{\mathrm{d} E_{p}^{2}}{\mathrm{~d} V}
$$

ou encore en :

$$
i(V)=\frac{\varepsilon_{0}}{2} p^{2} \frac{\mathrm{d}}{\mathrm{d} V} v_{d+} \frac{\mathrm{d} E_{p}^{2}}{\mathrm{~d} V} .
$$

Si la vitesse de dérive des ions est limitée par l'échange de charge,

$$
\begin{aligned}
& v_{d+}=v_{1} \sqrt{E_{p}}, \\
& i(V)=\frac{2}{5} \varepsilon_{0} p^{2} v_{1} \frac{\mathrm{d}^{2}}{\mathrm{~d} V^{2}} E_{p}^{5 / 2},
\end{aligned}
$$

et sous cette forme, on peut considérer des cas particuliers :

Entre $V_{\mathrm{c}}$ et $V_{\mathrm{c}}-V_{\mathrm{i}}$, il n'y a pas d'ionisation. Donc

$$
E_{p}^{5 / 2}=E_{p}^{5 / 2}\left(V_{\mathrm{c}}\right)-C\left(V_{\mathrm{c}}-V\right) .
$$

Si l'on suppose

$$
E_{p}^{2} \rightarrow b V^{k}
$$

$k=1$ correspondant à la variation linéaire de $E_{p}(x)$ qu'on observe expérimentalement.

$$
i(V)=\frac{k}{2}\left(\frac{5 k}{4}-1\right) \varepsilon_{0} p^{2} v_{1} b^{5 / 4} V^{\left(\frac{5 k}{4}-2\right)} .
$$

On voit donc que pour $k=1, i(V)$ varie comme $1 / V^{3 / 4}$ mais on voit aussi que si $i(V)$ est petit par rapport à l'ionisation produite dans la lueur négative $k=4 / 5$, ce qui n'est pas loin de l'observation expérimentale.

L'équation de Poisson sert d'abord à vérifier si la forme supposée pour la fonction $E_{p}^{2}(V)$ est correcte. Si celle-ci est satisfaisante, elle détermine le facteur de proportionnalité $J_{00}$ en fonction de $V_{\mathrm{c}}$.

4. Conclusion. - La solution de l'équation différentielle (5) pour la diffusion ambipolaire d'un plasma, qui tient compte d'une source homogène uniforme et de la recombinaison, est une fonction elliptique (14), et elle est indépendante d'un courant appliqué à divergence nulle. En particulier, il y a trois limites plus simples (19), (22), (25). Comme la théorie ambipolaire conduit à une distribution de Boltzmann, on connaît le potentiel ambipolaire (29), et en appliquant la formule usuelle pour le courant aléatoire, on obtient le potentiel flottant des parois (30), et un supplément (33) donne l'effet d'un courant continu $J$ très petit. Cette formule est semblable à celle d'une sonde double, mais comme les électrodes ne sont pas très proches, il faut ajouter un potentiel ohmique (34).

Les formules ci-dessus sont suffisantes si le courant $J$ est entièrement fourni par la source $S$, comme dans une chambre d'ionisation ; mais en pratique on utilise des courants beaucoup plus forts. Il se forme alors une chute cathodique suffisante pour que l'émission secondaire de la cathode fournisse le courant demandé, et il faut accoler une région cathodique à la région ambipolaire étudiée plus haut. La théorie conventionnelle de la chute cathodique fait usage du coefficient d'ionisation $\alpha_{i}$ de Townsend qui est fonction de la valeur locale de $E / p$. Or, l'existence même de la lueur négative montre que les électrons transportent l'énergie du potentiel de la cathode bien au-delà de la région de chute de potentiel.

Dans la deuxième partie, nous avons introduit (5), (6) des coefficients d'ionisation et d'excitation qui sont à la fois fonctions de $E / p$ et de l'énergie cinétique $u$ des électrons. Puis nous avons établi l'éq. (10) pour le flux des électrons dans l'espace des énergies cinétique et potentielle, ainsi que les conditions aux limites (19), (22), (23), et une condition de fermeture (33). Cette équation doit être résolue par ordinateur. On s'attend à ce que la distribution de l'ionisation soit assez différente de celle de la théorie classique, mais à ce que la chute de potentiel normale ne soit pas très différente. Il restera à calculer une région de transition dans laquelle la diffusion n'est pas négligeable, comme dans la chute cathodique, mais n'est quand même pas ambipolaire.

\section{Bibliographie}

[1] Fenstermacher, C. A., Nutter, M. J., RinK, J. P., Boyer, K., Bull. Am. Phys. Soc. 16 (1971) 42.

[2] Brown, S. C., Introduction to electrical discharges in gases (J. Wiley \& Sons Inc. New York) 1966.

[3] Lowke, J. J. and Davies, D. K., Scientific Paper 72-108ARCPL-P 4, Westinghouse Research Laboratories, Pittsburgh, Pennsylvania (1972).

[4] Little, P. F. et von Engel, A., Proc. R. Soc. A 224 (1954) 209.
[5] Hantzsche, E., Beitrage a. d. Plasma Physik 1 (1960-61) 179.

[6] KleIN, W., Wiss. Z. Tech. Hochsch., Iemenau 277 (1968) 127.

[7] Parker, A. B., Johnson, P. C., Proc. R. Soc. A 325 (1971) 511. Notre formule ne tient pas compte de la chute libre d'une partie des électrons.

[8] Tran Ngoc, A., Marode, E., Johnson, R. C., IEE Gas Discharges 9-17 Sept. London (1974).

[9] Davis, W. D., Vanderslice, T. A., Phys. Rev. 131 (1963) 219. 